\title{
Physical Phenomena in Z-pinch Plasma of Impulse Plasma Deposition Process
}

\author{
M. RABIŃsKI ${ }^{a, *}$ AND K. ZDUNEK ${ }^{b, \dagger}$ \\ ${ }^{a}$ Department of Plasma Physics and Technology
}

The Andrzej Sołtan Institute for Nuclear Studies, 05-400 Otwock-Świerk, Poland

${ }^{b}$ Faculty of Materials Science, Warsaw University of Technology

Wołoska 141, 02-507 Warsaw, Poland

\begin{abstract}
In the present paper we propose a model of physical phenomena behind the front face of the electrodes in an impulse plasma accelerator. The model is based on the results of recent experimental observations and measurements. It correlates plasma dynamics with mechanism of phenomena in a column of pinching plasma. On the contrary to the previous model the current one suggests the series of relatively short pulses of metallic ions from the erosion of electrode material. Till now the pinch was treated rather as a nearly continuous source of metallic plasma, feeding the process with ions from the erosion of electrode material.
\end{abstract}

PACS numbers: 07.05.Tp, 52.30.-q, 52.35.Py, 52.77.Dq

\section{Introduction}

The impulse plasma generated by an electric discharge is an efficient source of ions. When appropriately controlled by electric and magnetic fields, discharge chamber geometry and parameters, plasma can be used in surface engineering as a tool for synthesizing materials in the form of coatings deposited on different substrata.

During the impulse plasma deposition (IPD) process plasma is generated in the working gas due to a high-voltage high-current pulse discharge, ignited within a coaxial accelerator [1]. Schematic diagram of the IPD apparatus is shown in Fig. 1. A system of two coaxial electrodes (a cylinder and a rod, insulated from one another by a ceramic material), in which the plasma is accelerated by

*e-mail: rabinski@ipj.gov.pl

$\dagger_{\text {e-mail: zdunek@inmat.pw.edu.pl }}$ 
the Lorentz force, is the most efficient and commonly used solution for plasma engineering. At the outlet of the accelerator, plasma consists of the ionized atoms of the working gas and the eroded electrode material. In particular, to obtain titanium nitride coatings a titanium rod is used as the internal electrode and nitrogen as the working gas.

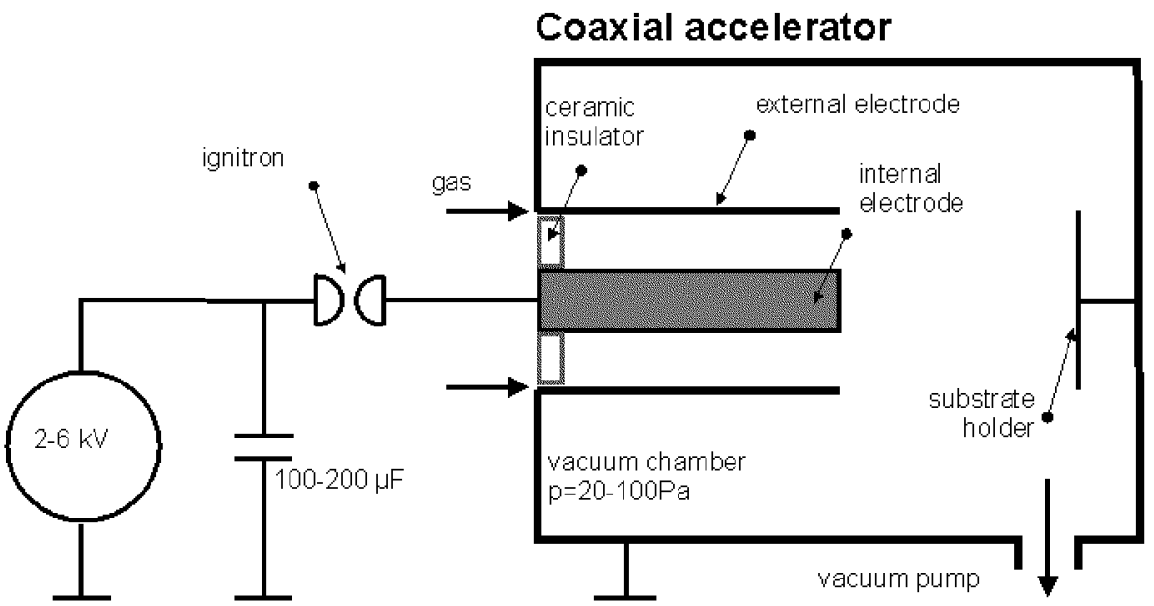

Fig. 1. Scheme of the impulse plasma coaxial accelerator.

Theoretical models and computational studies have significantly increased the understanding of the IPD process. In our earlier studies [2] we worked out a physical model of dynamic phenomena during a coaxial phase of IPD discharge. There has been also, however, a continually growing demand for validation of plasma dynamics code by comparison with relevant experimental observations. Therefore, a special programme was started $[3,4]$ to investigate plasma dynamics with the help of high-speed photography, X-ray and ion beam diagnostics.

In the present paper we report physical phenomena in a z-pinch area behind the front face of the accelerator electrodes. This model is based on the results of recent experimental observations and measurements $[5,6]$. It correlates plasma dynamics with phenomena in a column of pinching plasma.

\section{Experimental studies of $z$-pinch region}

The structure of the current sheet, as well as dynamics of its spreading, has been examined experimentally in a visible spectrum with the high-speed frame cameras (HSFCs) [5]. In the following figures (see Fig. 2) the appropriate plasma images obtained during the current first half-period of accelerator work are presented. The specific tilt of discharge symmetry axis towards bottom of observed region is caused by the diagnostic windows cut in an upper part of the outer electrode. Plasma outflow through these windows relieves the moving current sheet 
of swept gas and accelerates motion in the upper side of the discharge chamber. Thus plasma from this region overtakes in evolution plasma from the unmodified part of accelerator on the bottom of the photograph.

$t=11.50 \mu \mathrm{s}$
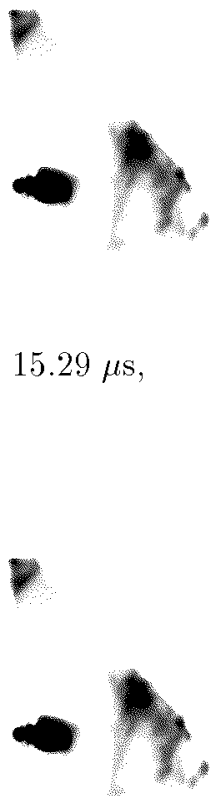

$t=13.40 \mu \mathrm{s}$, $t=13.44 \mu \mathrm{s}$ $t=15.29 \mu \mathrm{s}$

$t=19.44 \mu \mathrm{s}$,

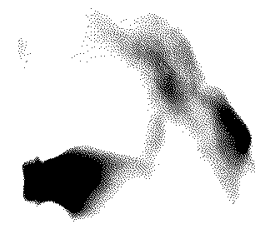

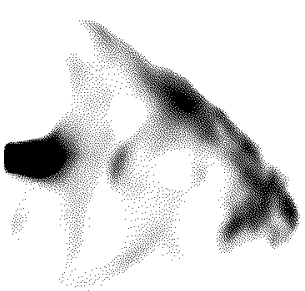

$t=21.43 \mu \mathrm{s}$

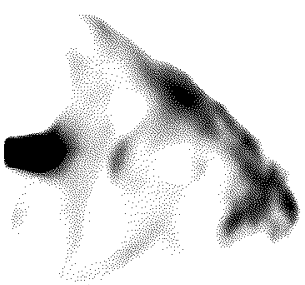

Fig. 2. High-speed images in visible spectrum of plasma at the IPD accelerator outlet; $U=6 \mathrm{kV}, C=100 \mu \mathrm{F}$, working gas - nitrogen at $p=60 \mathrm{~Pa}$, electrode front-end on the left side of the image, image width $-8.5 \mathrm{~cm}$.

One can observe the characteristic features of the $z$-pinch region behind the front face of the accelerator electrodes:

- The dense plasmoid as the result of plasma sweeping on the current sheet surface in the region of the Raleigh-Taylor instability;

- Pinching of the plasma column.

The emission of $\mathrm{X}$-rays and ion beams have been examined experimentally with the help of XET (X-ray energy in time) detector and Faraday Cup [6]. These signals have been correlated with total discharge current time derivative from the Rogowski coil. The presence of simultaneous emissions of X-rays and ion beams has been proved. These emissions probably are correlated with plasma column pinches - unfortunately, the high-speed photographs and the emission diagnostic were taken during different experimental sessions. 


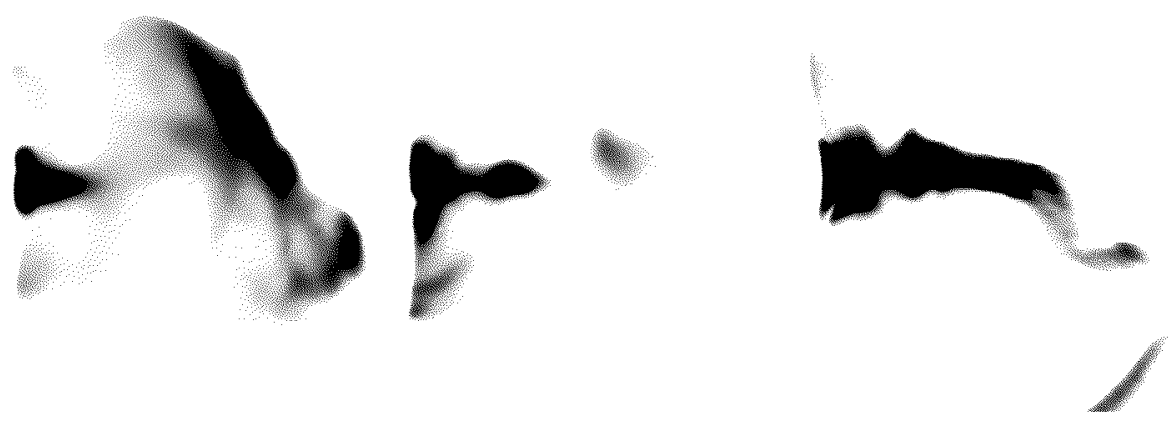

Fig. 3. Experimental results of X-ray and ion beam diagnostics; markers on horizontal time scale $-10 \mu \mathrm{s} /$ div, signals in the channels (from top to bottom) - electric current as measured with the Rogowski coil, ion beam diagnostic with Faraday Cup, hard X-rays from XET detector, soft X-rays $\sim 2 \mathrm{keV}$.

After $15 \mu$ s from discharge ignition one can observe a few to more than ten sudden oscillations of electric current (see Fig. 3). Each oscillation has a wa velength of about $0.1-0.5 \mu \mathrm{s}$, the mean distance between pulses equals $2.0-2.5 \mu \mathrm{s}$ while the duration of oscillation period reaches about $20 \mu \mathrm{s}$. The situation repeats during the second half-period after the change of electrode system polarization. The observed timing strictly correlates with stages of IPD accelerator discharge predicted by the theoretical model $[2,3]$ previously worked out and with plasma images from the high-speed frame camera.

\section{Physical phenomena in $z$-pinch plasma}

Results of X-ray and ion beam emission observations, as well as the frame camera images lead to the following model of the physical phenomena during pinching of plasma column:

- The $z$-pinch of the IPD process looks much like an arc discharge column with some so-called "sausage" instabilities, separated from one another in space and time. The regions of this instability looks like a narrowing of plasma column. Plasma pinching in the narrowings causes the emission of soft X-rays $(\sim 3 \mathrm{keV})$ and ion beams with kinetic energy of nitrogen ions estimated for $10-15 \mathrm{eV}$.

- The duration of the $z$-pinch phase reaches about $20 \mu \mathrm{s}$. Thus the nature of the IPD pinch significantly differs from the typical $z$-pinch in the Plasma-Focus device, dominated by the numerous sausage instabilities. 
- In the $z$-pinch column a beam of energetic electrons is accelerated toward the internal electrode front end. This phenomenon effects in a characteristic funnel-like erosion of electrode material. Diagram of erosion mechanism of this type is shown in Fig. 4. Such erosion has been observed even in the case of aluminium oxide insert [4], which is a very good dielectric.

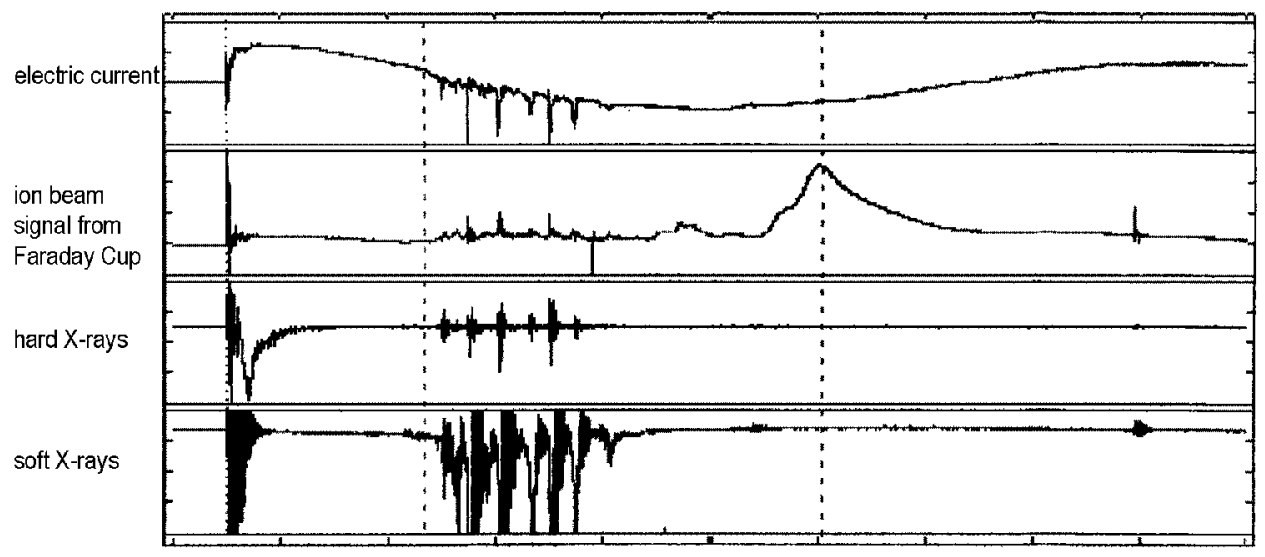

Fig. 4. Model of electron motion in the region of internal electrode free end with the ceramic insert.

The presented findings increase the understanding of the IPD process and thus the deposition of coatings on substrate surface. Till now the pinch was treated rather as a nearly continuous source of metallic plasma, feeding the process with ions from the erosion of electrode material. On the contrary to the previous model the results of recent experimental observations and measurements suggest the series of relatively short pulses of ions.

\section{Acknowledgment}

This work was supported by the State Committee for Scientific Research under the contract KBN 7 T08C 05417.

\section{References}

[1] K. Zdunek, J. Mater. Sci. 26, 4433 (1991).

[2] M. Rabiński, K. Zdunek, Vacuum 48, 715 (1997).

[3] M. Rabiński, K. Zdunek, Acta Phys. Pol. A 96, 319 (1999).

[4] M. Rabiński, K. Zdunek, Surf. Coat. Techn. 116-119, 679 (1999).

[5] M. Rabiński, K. Zdunek, M. Paduch, K. Tomaszewski, Surf. Coat. Techn. 142-144, 49 (2001).

[6] J. Baranowski, L. Jakubowski, M. Rabiński, K. Zdunek, submitted to Vacuum. 